\title{
Mapping of folic acid in the children brainstem
}

\author{
Ewing Duque-Díaz ${ }^{1}$, Rafael Coveñas ${ }^{2,3}$ \\ ${ }^{1}$ Laboratory of Neurosciences, School of Medicine, Universidad de Santander, Bucaramanga, Colombia, ${ }^{2}$ Institute of Neurosciences of Castilla y León \\ (INCYL), Laboratory of Neuroanatomy of the Peptidergic Systems, University of Salamanca, Salamanca, ${ }^{3}$ Grupo GIR BMD (Bases Moleculares del \\ Desarrollo), University of Salamanca, Salamanca, Spain
}

\begin{abstract}
Using highly specific antisera, the neuroanatomical distribution of folic acid (FA) and retinoic acid (RA) has been studied for the first time in the children brainstem. Neither immunoreactive structures containing RA nor immunoreactive fibers containing FA were found. FA-immunoreactive perikarya (fusiform, small/medium in size, one short dendrite) were only found in the pons in three regions: central gray, reticular formation, and locus coeruleus. The number of cell bodies decreased with age. In the first case studied ( 2 years), a moderate density of cell bodies was observed in the central gray and reticular formation, whereas a low density was found in the locus coeruleus. In the second case (6 years), a low density of these perikarya was observed in the central gray, reticular formation, and locus coeruleus. In the third case (7 years), a low density of FA-immunoreactive cell bodies was found in the central gray and reticular formation, whereas in the locus coeruleus no immunoreactive cell bodies were observed. The distribution of FA in the central nervous system of humans and monkeys is different and, in addition, in these species the vitamin was located in different parts of the nerve cells. The restricted distribution of FA suggests that the vitamin is involved in specific physiological mechanisms.
\end{abstract}

Key words: Folic acid, Humans, Immunohistochemistry, Retinoic acid, Vitamins

Received January 31, 2021; Revised April 19, 2021; Accepted April 21, 2021

\section{Introduction}

Vitamins are complex structures with a variety of functions and, in nature, show a wide distribution. Although the metabolic role played by vitamins (acting as coenzymes or cofactors) in many reactions is well known $[1,2]$, the knowledge on the neuroanatomical localization of these molecules in the mammalian brain is lesser. In recent years, due to the development of new highly specific antibodies against vitamins, the neuroanatomical distribution of these compounds in the mammalian central nervous system has become a promising line of research [3-10]. In this sense, the distribution of fibers and cell bodies containing folic acid

\section{Corresponding author:}

Ewing Duque-Díaz (iD)

Laboratory of Neurosciences, School of Medicine, Universidad de Santander, Bucaramanga 680003, Colombia

E-mail:ew.duque@mail.udes.edu.co
(FA), retinoic acid (RA) (a metabolite of retinol or vitamin A), thiamine (vitamin $B_{1}$ ), riboflavin (vitamin $B_{2}$ ), nicotinamide (the amide of nicotinic acid or vitamin $B_{3}$ ), pantothenic acid (vitamin $\mathrm{B}_{5}$ ), pyridoxal/pyridoxine (natural forms of vitamin $\mathrm{B}_{6}$ ) or vitamin $\mathrm{C}$ has been reported in the brain of rats, monkeys and humans. Most of the previous studies were performed in the central nervous system of monkeys [3-9, 11]. The knowledge on the anatomical distribution of vitamins leads to gain insight into the role played by these compounds (e.g. excitatory and/or neurotrophic actions) in the mammalian central nervous system regions in which the presence of vitamins is found. The neuroanatomical distribution of FA and RA has been respectively reported in the monkey and rat brains $[6,10]$ and vitamin $C$ has been the only vitamin studied to date in humans (children brainstem) [5]. Thus, currently no study on the neuroanatomical distribution of both FA and RA has been performed in the human central nervous system.

FA acts as cofactor/coenzyme (e.g. involved in the biosyn- 
thesis of thymidylate, methionine and purine nucleotides, in the reduction/oxidation of single carbon units), it is widely distributed in foods and its intake (high concentration) can prevent defects in the neural tube, vascular diseases and cancer and, in addition, FA has been involved in the proliferation/apoptosis of astrocytes and in neurodegenerative diseases (e.g. Parkinson, Alzheimer) [6, 12-16]. In Alzheimer's disease, FA dietary deficiency affected the accumulation of $\beta$-amyloid $[17,18]$, whereas in Parkinson's disease the intake of FA promoted a neuroprotective effect $[19,20]$. In the monkey brain [6], only immunoreactive fibers containing FA were located in the brainstem and thalamus and the authors of this study suggested that FA could be involved in somatosensorial, motor, visual and auditory mechanisms. Thus, although it must be confirmed in future studies, it seems that according to the neuroanatomical studies performed in the mammalian brain vitamins could be involved in mechanisms other than pure metabolic actions. Regarding the RA, this vitamin is synthesized from retinol (vitamin A), which in turn is required by vertebrates for normal tissue development and maintenance [21-24]. In mammals, the distribution of RA has been indirectly detected by demonstrating the presence of RA-synthesizing enzymes (retinaldehyde dehydrogenases); however, in 2012, an antiserum directed against RA was developed by our group and the presence of RA was directly detected by using immunohistochemical methods in the rat brain [10]. In this study, only cell bodies containing RA were detected in the hypothalamus. In addition, by using high performance liquid chromatography this vitamin has been also detected in the hippocampus, striatum, cerebellum, testis, kidney and liver [25,26].

According to the data reported above, no information is currently available on the neuroanatomical distribution of FA and RA in the human brain. Thus, in order to increase our knowledge about this distribution our aim is to study by using immunohistochemical techniques the distribution of both vitamins in humans. The results of this study will serve to gain information about the possible unsuspected physiological actions mediated by both vitamins.

\section{Materials and Methods}

The experimental design, procedures and protocols of this work have been performed under the guidelines of the ethic and legal recommendations of the Colombian legislation (Resol. 8430/1993) and in accordance with the Helsinki dec- laration. Furthermore, this work was approved by the Ethic Committee of the University of Santander (UDES, Bucaramanga, Colombia) (Act 023-17). In this study, three brains of male children (2, 6, and 7 years old) who died respectively from respiratory failure, dengue, and drowning by immersion were used. According to the Colombian legislation brains were collected, from routine autopsies, within 24-48 hours after death (University of Santander, Bucaramanga, Colombia). In all cases, the pathological reports showed no evidence of neurological diseases.

The brain of each subject was removed and later the brainstem was dissected out. As previously described [5], brainstems were immersed in 10\% formalin (three weeks at $4^{\circ} \mathrm{C}$ ) to keep them preserved and then transferred to $4 \%$ formaldehyde in $0.1 \mathrm{M}$ phosphate buffer-saline (PBS; $\mathrm{pH}$ 7.4) (for 30 days at $4^{\circ} \mathrm{C}$ ). After fixation, brainstems were kept in PBS at $4^{\circ} \mathrm{C}$ and cryoprotected in increasing sucrose solutions (5\%-30\%) until they sank. Brainstems were cut using a cryostat (Leica CM1860): $50 \mu \mathrm{m}$ thick coronal sections were taken, collected in PBS, kept at $4^{\circ} \mathrm{C}$, and processed for immunostaining. One-in-six sections were mounted onto adhesive slides for Nissl staining.

\section{Immunohistochemistry}

As previously described [5, 6, 10, 27], free floating sections were treated with distilled water containing $\mathrm{H}_{2} \mathrm{O}_{2}$ (30\%), $\mathrm{NH}_{3}(20 \%)$ and $\mathrm{NaOH}$ (1\%) for 20 minutes in order to avoid interference with endogenous peroxidases [28]. Sections were washed for 20 minutes in PBS (0.15 M; pH 7.2) and pre-incubated for 30 minutes in PBS containing Triton X-100 (0.3\%) and normal horse serum (2\%). Sections were incubated overnight $\left(4^{\circ} \mathrm{C}\right)$ in the above mixture containing the anti-FA antiserum (diluted 1:500) or the anti-RA antiserum (diluted 1:500). Following this, sections were washed in PBS (30 minutes) and incubated at room temperature with biotinylated anti-rat (FA) or anti-rabbit (RA) immunogammaglobulins (Vector; Vector Laboratories, Burlingame, CA, USA) diluted $1 / 200$ in PBS (60 minutes). Then, sections were washed in PBS (30 minutes), incubated for 60 minutes with avidinbiotin-peroxidase complex (Vectastain, Vectastain standard ABC kit, diluted 1/100; Vector Laboratories), washed in PBS (30 minutes) and Tris- $\mathrm{HCl}$ buffer ( $\mathrm{pH}$ 7.6; 15 minutes) and the tissue-bound peroxidase was developed with $\mathrm{H}_{2} \mathrm{O}_{2}$ using 3, 3' diaminobenzidine as chromogen. Finally, sections were rinsed with PBS, dehydrated and coverslipped with Entellan. In addition, in brainstem sections the heat-induced epi- 
tope retrieval (HIER) method was performed [29]. Thus, a container with Tris-EDTA recovery buffer (10 nM Tris, 1 mM EDTA, $0.05 \%$ Triton X-100, $\mathrm{pH} 9.0$ ) was preheated in a rice cooker with water until reaching $97^{\circ} \mathrm{C}$. Then, sections were carefully soaked in the buffer for 40 minutes. Later, the container with the samples was removed and the tap water was allowed to run for about 10 minutes until it cools. Finally, sections were removed and immersed in PBS until the immunohistochemistry technique was performed.

\section{Specificity of the antisera}

The immunological properties of the polyclonal primary FA and RA antisera used here have been reported previously in monkeys (FA) [6] and rats (RA) [10]. Both antibodies were purchased from commercial sources (Gemacbio S.A., Saint Jean d'Illac, France) and were raised in rats (FA, reference AP099) and rabbits (RA, reference: AP057) with their respective bovine serum albumin (BSA) immunogens. Thus, rats/rabbits were immunized by one injection every 2-3 weeks. Each administration (subcutaneous injection) contained a mixture of $250 \mu \mathrm{l}$ of complete (only used in the first immunization)/incomplete Freund adjuvant and $250 \mu \mathrm{l}$ of an immunogenic $\mathrm{NaCl}$ solution $[4,8]$. Serum samples were collected every three weeks and the antisera were pre-purified by immunoabsorption and precipitated. Later, the antibodies raised were characterized in ELISA tests $[6,10]$. In both cases (FA and RA), the antibody avidity $\left(\mathrm{IC}_{50}\right)$ was rather high $\left(10^{-8} \mathrm{M}\right)$.

As previously reported $[6,10]$ and in order to prevent nonspecific immunoreactivity due to the anti-carrier antibodies, the first antiserum was preabsorbed (before the immunohistochemical application) with the respective coupling agent and carrier protein. Moreover, as previously reported [6], the specificity of the anti-FA antibody was very high, since the antiserum discriminated conjugated FA from other conjugated molecules (e.g. tetrahydrofolic acid, riboflavin, folinic acid, methotrexate, BSA) very well. This also occurs for the anti-RA antibody, since the antiserum discriminated conjugated RA from other conjugates molecules (e.g. trans-farnesyl-cysteine, palmitic acid, myristic acid, oleic acid, BSA) [10]. In the present study, the specificity of the immunostaining was in addition controlled by omitting the FA antiserum in the first incubation bath (no residual immunoreactivity was observed) and by treating the FA antiserum with an excess $(100 \mathrm{mg} / \mathrm{ml}$ diluted antiserum) of synthetic FA (no immunoreactivity was also observed).

\section{Mapping}

According to previous works [5,27,30], the atlas of Haines (2012) [31] was used for mapping and nomenclature. One out of six sections was routinely stained for Nissl substance with cresyl violet to delineate the brainstem nuclei in which the immunoreactivity was observed. To determine the density of the immunoreactive cell bodies in the children brainstem, perikarya were graded into three categories as previously reported [27]: high (more than 20 cell bodies/region/section), moderate (10-20 cell bodies /region/section) and low (less than 10 cell bodies/region/section). According to the size of the cell body and, as previously described [27], immunoreactive cell bodies were considered large $(>25 \mu \mathrm{m}$ in diameter), medium-size $(15-25 \mu \mathrm{m})$ and small $(<15 \mu \mathrm{m})$. Photomicrographs were obtained with an Olympus DP21 digital camera attached to an Olympus BX43 microscope (Olympus, Tokyo, Japan). To improve the visualization of the results, only the contrast and brightness of the images were adjusted (Adobe Photoshop CS6 Software; Adobe, San Jose, CA, USA), without any further manipulation of the photographs.

\section{Cell count}

To determine the number and average of immunoreactive cell bodies, images were digitized according to the imageJ software (developed by the NIH) and available free of charge on internet [32]. The colour images for each case $(2,6$, and 7 years) were loaded into the imageJ software and a duplicate was made to work on it. Then, a scale adjustment was made according to the objective with which the images were captured to calibrate the section area. Following this, shadows were eliminated and the region to be treated was delimited. The photographs were converted into an 8-bit image type, followed by a threshold adjustment and binarization so that only the counting points remained. Finally, a particle analysis was carried out, giving the sum and average of perikarya.

\section{Results}

Neither immunoreactive structures containing RA (fibers or cell bodies) nor FA-immunoreactive fibers were observed in the children brainstem. Thus, only cell bodies containing FA were found and they were exclusively located in the pons at the level showed in Fig. 1. It is important to remark that no difference was observed in the distribution of cell bodies containing FA in the children brainstem when the HIER method was applied or not. In addition, after applying 


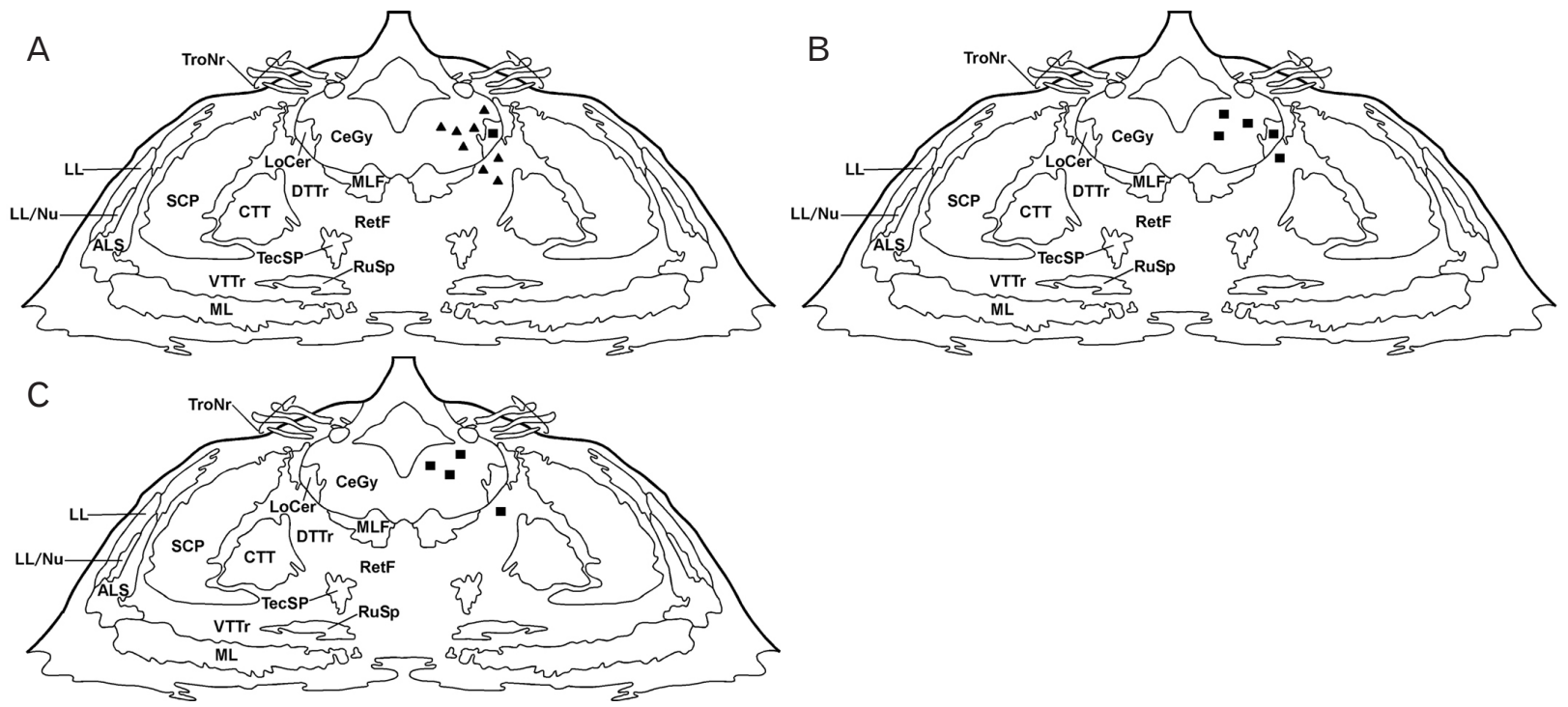

Fig. 1. Distribution of folic acid (FA)-immunoreactive cell bodies in frontal planes of the human pons. Two (A), six (B), and seven years (C). Cell bodies are represented by closed triangles (moderate density) and closed squares (low density). ALS, anterolateral system; CeGy, central gray; CTT, central tegmental tract; DTTr, dorsal trigeminothalamic tract; LL, lateral lemniscus; LL/Nu, lateral lemniscus nucleus; LoCer, locus coeruleus; ML, medial lemniscus; MLF, medial longitudinal fasciculus; SCP, superior cerebellar peduncle; RetF, reticular formation; RuSp, rubrospinal tract; TecSp, tectospinal tract; TroNr, trochlear nerve, VTTr, ventral trigeminothalamic tract.

Table 1. Folic acid-immunoreactive cell bodies in the children brainstem

\begin{tabular}{|c|c|c|c|c|c|c|c|c|c|}
\hline \multirow[b]{2}{*}{ Nuclei } & \multicolumn{3}{|c|}{2 years } & \multicolumn{3}{|c|}{6 years } & \multicolumn{3}{|c|}{7 years } \\
\hline & Total no. & $\begin{array}{c}\text { Mean ir CB/ } \\
\text { section }\end{array}$ & Density & Total no. & $\begin{array}{c}\text { Mean ir CB/ } \\
\text { section }\end{array}$ & Density & Total no. & $\begin{array}{c}\text { Mean ir CB/ } \\
\text { section }\end{array}$ & Density \\
\hline GeGy & 117 & 14.625 & ++ & 47 & 5.875 & + & 14 & 1.750 & + \\
\hline RetF & 87 & 10.75 & ++ & 34 & 4.25 & + & 1 & 0.125 & + \\
\hline LoCer & 12 & 1.50 & + & 8 & 1.00 & + & 0 & 0.00 & - \\
\hline
\end{tabular}

ir, immunoreactive; CB, cell bodies; GeGy, central gray (pons); RetF, reticular formation (pons); LoCer, locus coeruleus. In all cases, eight sections were studied.

this method, no immunoreactive structure containing RA was visualized. In all cases, cell bodies containing FA were fusiform, small/medium in size and showed in general one short dendrite. In addition, FA-immunoreactive cells bodies showed a restricted distribution: they were only observed in the central gray, locus coeruleus and reticular formation (above the dorsal trigeminothalamic tract). It is important to note that the number of the immunoreactive cell bodies varied depending on the age (Table 1). Thus, it was observed that as the age of the subjects increased, the number of the immunoreactive-cell bodies decreased (Figs. 1, 2A-C, 3); however, in the three cases studied the intensity of the immunoreactivity was similar.

In the first case ( 2 years), a moderate density of cell bodies was observed in the central gray (Figs. 1A, 2A, 3A, B) and in the reticular formation located above the dorsal trigeminothalamic tract (Fig. 2A), whereas a low density was found in the locus coeruleus (Figs. 2A, 4A). In the second case (6 years), a low density of these perikarya was observed in the central gray, above the dorsal trigeminothalamic tract (reticular formation) and in the locus coeruleus (Figs. 1B, 2B, $3 \mathrm{C}, \mathrm{D}, 4 \mathrm{~B})$. In the third case (7 years), a low density of FA-immunoreactive cell bodies was found in the central gray (Figs. $1 \mathrm{C}, 2 \mathrm{C}, 3 \mathrm{E}, \mathrm{F}$ ) and reticular formation, whereas in the locus coeruleus no immunoreactive cell bodies were observed.

Table 1 shows the number and density of cell bodies containing FA in the human brainstem. Thus, the digital analysis of images obtained (eight for each age) showed that for the two-year-old subject, the total number of immunoreactive cell bodies in the central gray was 117 (mean 14.625), while for the reticular formation and locus coeruleus was 87 (mean 10.75) and 12 (mean 1.50), respectively. For the six-yearold subject, the total number of immunoreactive perikarya observed was 47 (mean 5.875) in the central gray, 34 (mean 


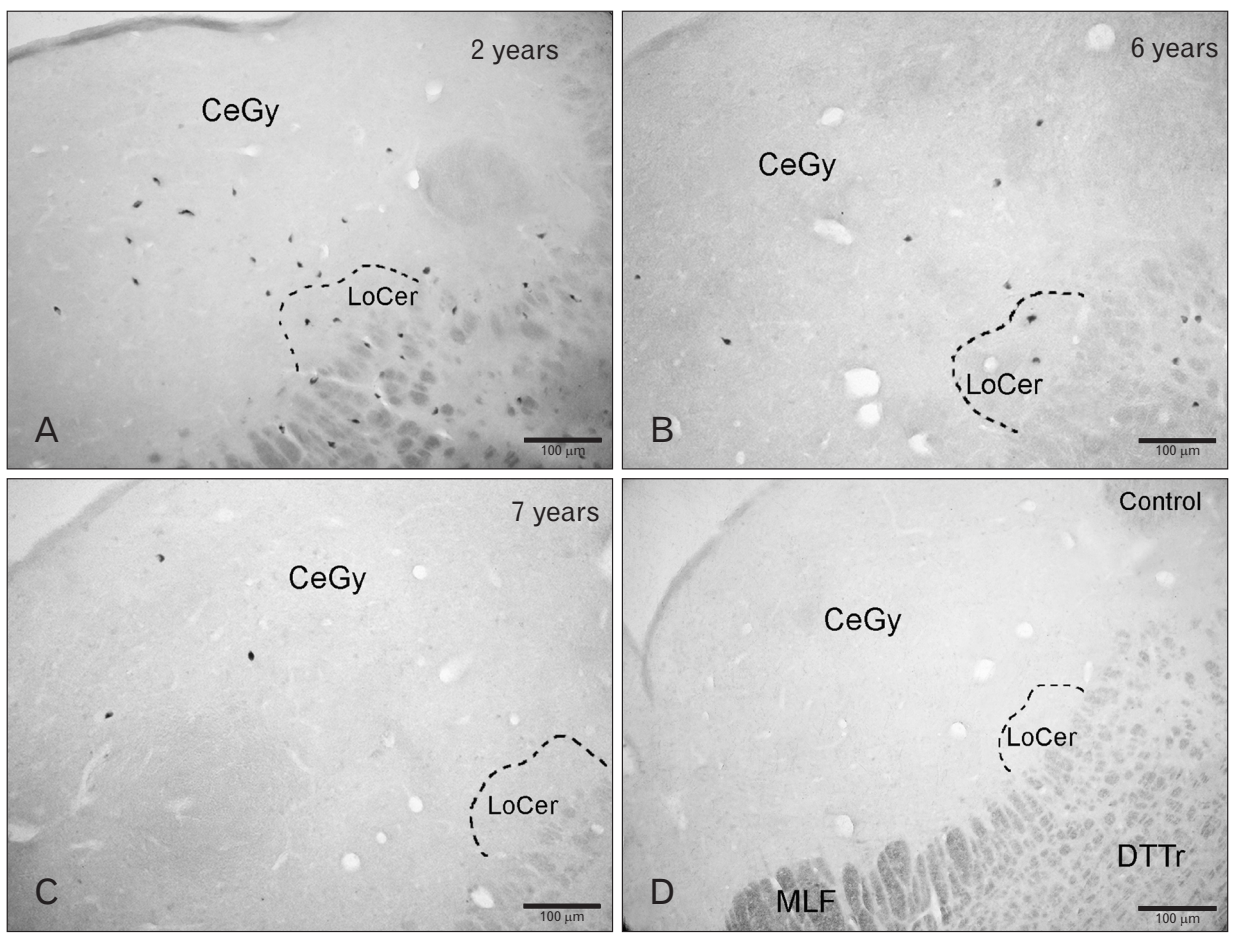

Fig. 2. Folic acid (FA) in the children pons. (A) Cell bodies containing FA in the central gray (CeGy), reticular formation (RetF), and locus coeruleus (LoCer) (2 years) (A) and 6 years (B). (C) Immunoreactive cell bodies in the CeGy (7 years). (D) Histological control (preabsorption of the anti-FA with an excess of synthetic FA). DTTr, dorsal trigeminothalamic tract; MLF, medial longitudinal fasciculus.
4.25 ) in the reticular formation and 8 (mean 1.00) in the locus coeruleus. Finally, for the seven-year-old subject, a total number of 14 (mean 1.750) immunoreactive cell bodies were observed in the central gray, whereas in the reticular formation 1 (mean 0.125) immunoreactive cell body was observed. At this age, no immunolabelled cell bodies were observed in the locus coeruleus.

\section{Discussion}

According to the neuroanatomical distribution of vitamins in the mammalian brain, it has been suggested that these compounds could play more important actions than their well-known metabolic functions [3-6, 10]. Thus, studies focused on the neuroanatomical distribution of vitamins in the mammalian central nervous system are important because, according to this distribution, unexpected actions of these compounds could be discovered. Here, the mapping of FA in the human brainstem is described for the first time. Immunohistochemistry is a suitable tool to increase the knowledge on the distribution of vitamins in the mammalian central nervous system and, in the future, the neuroanatomical findings reported here will contribute to know the physiological functions in which FA is involved in the human pons.
In the last decade, the mapping of immunoreactive structures containing vitamins (e.g. FA, RA, thiamine, riboflavin, vitamin $\mathrm{C}$ ) has been carried out by using highly specific antisera. These works were performed in rats $[10,11]$, monkeys [3, 4, 6-9] and humans [5]. The presence of immunoreactive fibers containing FA, but not cell bodies, has previously been reported in the adult monkey central nervous system in the following nuclei/regions: geniculate, pulvinar, ventral posteromedial, parafascicular, dorsal mesencephalon and medial thalamus [6]. However, in children, we observed the presence of cell bodies in only three pontine regions (central gray, reticular formation, locus coerulus); regions in which FA immunoreactivity was not observed in monkeys. Thus, the distribution of FA in the central nervous system of monkeys and children is different and, in addition, in these species the vitamin was observed in different parts of the nerve cells. Currently, the reason for the cellular distribution of FA in humans (cell bodies) and monkeys (fibers) is unknown. In both species, the distribution of FA-immunoreactive structures is very restricted, although the distribution is more widespread in monkeys than in humans. In general, except in the case of vitamin C (humans), the distribution of other studied vitamins (e.g. pantothenic acid, thiamine, riboflavin, pyridoxal) is restricted in the mammalian central nervous system or even a lack of immunoreactivity for some 


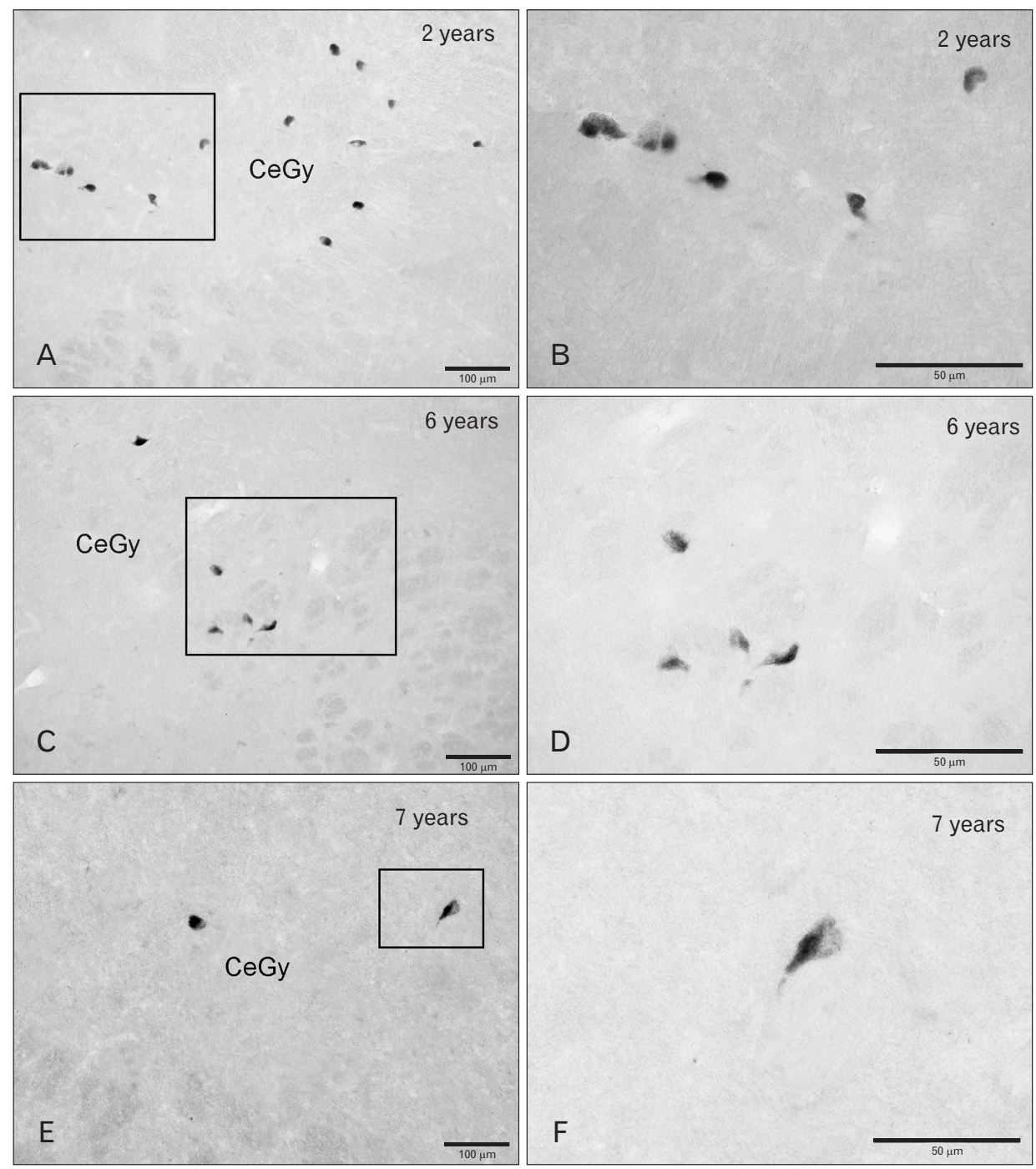

Fig. 3. Folic acid (FA)-immunoreacive cell bodies in the children pons central gray (CeGy). Two (A), six (C), and seven (E) years. (B, D, F) Higher magnifications of the regions delimited by rectangles in $\mathrm{A}, \mathrm{C}$, and $\mathrm{E}$.
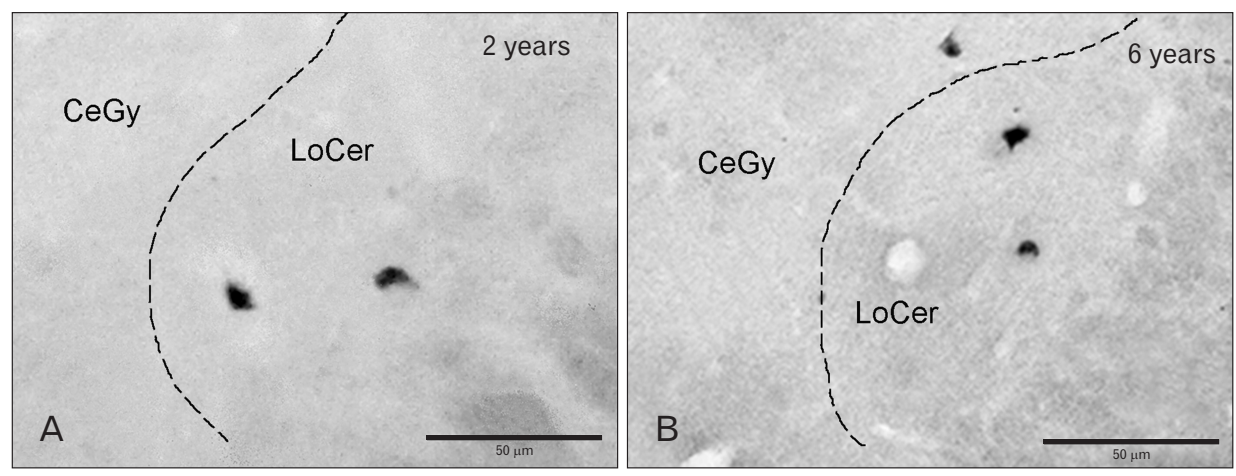

Fig. 4. Folic acid (FA)-immunoreactive perikarya in the children pons. (A) Locus coeruleus (LoCer) (2 years). (B) LoCer and central gray (CeGy) (6 years).

vitamins (e.g. pyridoxine, nicotinamide) has been reported $[7-9,11]$. Here, we did not observe immunoreactivity for RA; however, in rats, the presence of cell bodies containing this vitamin has been demonstrated in two hypothalamic regions (paraventricular hypothalamic nucleus, perifornical region)
[10]. In the latter study, no immunoreactive fibers containing RA were found. It is important to note that in the studies performed in rats (RA) [10], monkeys (FA) [6] and here in children (FA, RA), the same primary antisera and the same immunohistochemical procedure were used and applied re- 
spectively. Thus, to date, it seems that the distribution of FA and RA in the mammalian central nervous system is different in the species studied and because, except perfusion, the same methodological procedure (e.g. immunohistochemical technique, first antisera used) was applied, it seems that the differences found regarding the neuroanatomical distribution of FA and RA in the central nervous system of mammals are due to species differences. This must be confirmed in future studies. Although in general the distribution of immunoreactive fibers and cell bodies containing vitamins in the mammalian central nervous system is restricted $[3,4$, 6-11], it is important to note that the restricted distribution of the FA and the lack of immunoreactivity for RA in the children brainstem could be due to methodological considerations (e.g. cause of death, fixation procedure) that can mask the epitopes and decrease the binding with the FA/RA antisera, reducing the intensity/number of the immunoreactive structures. However, it is also important to remark that the distribution of FA-immunoreactive cell bodies observed in the children brainstem was the same after applying or not the HIER procedure. In addition, after applying this procedure, no immunoreactive structure containing RA was observed. The post-mortem delay (24-48 hours) could explain the absence of immunoreactive fibers containing FA/ RA in the children brainstem, the restricted distribution of perikarya containing FA and the absence of RA-immunoreactive cell bodies. However, in other studies using perfused experimental animals and in which the post-mortem time was minimal, the absence of fibers containing vitamins (e.g. vitamin $\mathrm{C}, \mathrm{RA}$ ) was reported in the mammalian central nervous system; in these cases, as in the present study, the immunoreactivity was exclusively observed in perikarya which showed a very restricted distribution. Other possibility could be that the immunohistochemical technique used here is insufficiently sensitive to visualize all the FA/RA containing profiles because they contain a very low level of both vitamins. It is also important to note that in our study, after the post-mortem period, cell structure was well maintained. This is in agreement with other studies performed on the distribution of neuropeptides in the human central nervous system $[33,34]$, in which both post-mortem period (24-48 hours) and experimental procedure (e.g., fixation, immunohistochemistry) were identical to those performed in the present study. Thus, in humans and using the same experimental protocol, the distributions of neuropeptides and vitamins were widespread and restricted respectively. In addition, in the three cases studied here the same distribution of FA-immunoreactive cell bodies was observed but the density of these cell bodies was different (the number of cell bodies decreased with age). Thus, altogether the data suggest that the perfusion time after death (24-48 hours) did not affect the distribution of vitamins (FA, RA) in the children brainstem. However, a decrease in the immunoreactivity for vitamins due to the post-mortem period (24-48 hours) must not be discarded.

To date, only one study has reported the neuroanatomical distribution of a vitamin in humans [5]; in this case, the distribution of vitamin $\mathrm{C}$ was performed in the brainstem of children during postnatal development [5]. In the latter study two groups of ages (younger and older than one year of life) were compared. Like here, immunoreactivity for vitamin $\mathrm{C}$ was only found in cell bodies, but the distribution/number of these perikarya was more widespread in older children and this means that the ability to retain vitamin $\mathrm{C}$ is maintained or increased with age. However, the opposite effect was observed for FA: the number of neurons containing FA was higher in younger children. Although, due to the low number of cases studied here (one per age), the latter observation must be confirmed in future studies. Moreover, the distribution of cell bodies containing vitamin $\mathrm{C}$ was widespread in the brainstem of children, whereas in the same region of the central nervous system the distribution of FA-immunoreactive perikarya was very restricted. In fact, to date, in the children brainstem vitamin $\mathrm{C}$ showed the most widespread distribution of a vitamin in the mammalian central nervous system. It is important to note that cell bodies containing vitamin $\mathrm{C}$ were observed in the three pontine regions in which FA-immunoreactive perikarya were observed; thus, a colocalization of cell bodies containing FA or vitamin $\mathrm{C}$ occurs. According to the morphological characteristics of the immunoreactive perikarya (e.g. one short dendrite, size), it seems that FA and vitamin $\mathrm{C}$ are located in the same cell bodies placed, for example, in the pontine central gray; however, the possible coexistence of both vitamins in nerve cells must be confirmed in future studies. Another important observation is that, in general, the neuroanatomical distribution of vitamins in the central nervous system of mammals is different and it seems that the distributions of those fibers/perikarya containing vitamins are exclusively due to the different patterns of distribution of these vitamins. It is important to note that in the case of the distribution of vitamin $\mathrm{C}$ in the monkey brain, cell bodies containing this vitamin was only found 
in the somatosensorial cortex (precentral gyrus); thus, the neuroanatomical distribution of vitamin $\mathrm{C}$ is quite different in monkeys and humans as it has been also mentioned above regarding the distribution of FA in both species.

The specificity of the immunoreactivity observed here, in addition to the histological controls performed, has previously been confirmed by using other techniques (e.g. ELISA) $[6,10]$. Applying the latter method, antibody specificity and avidity were confirmed. The protein carrier-coupling agent was considered as a competitor to avoid background and this means that the immunoreactivity observed for FA is specific and that it has nothing to do with the methodological protocol or with the previous processing of the tissue. Thus, the FA antiserum recognizes the target in a very specific manner because the antiserum shows a low cross-reactivity with molecules with similar structures (e.g. tetrahydrofolic acid, folinic acid) [6]. FA antiserum was pre-purified by immunoabsorption and no change in the specificity of the antibody was found as a result of this process.

Currently, the functional and specific roles of FA in the children brainstem are unknown. Thus, more neuroanatomical and physiological studies are required to know the functional roles played by FA; however, the restricted distribution of the vitamin in the children brainstem suggests that FA is involved in specific physiological actions which may vary according to the nuclei/regions in which this vitamin was located. For example, the presence of FA in the locus coeruleus suggests that the vitamin could be involved in the modulation of catecholaminergic function (e.g. synthesis of noradrenaline, at least during the first years of life) $[5,27]$. The antioxidant properties of FA have been linked to the prevention of various disorders and it is known that the development of the vertebrate central nervous system depends on FA optimal levels because the vitamin prevents neural tube defects and for this reason a diet containing FA is required for a normal brain function [35]. In humans, FA deficiency has been associated with neural tube defects (spina bifida, anencephaly, encephalocele) during early embryonic development [36]. These defects are induced by the monoubiquitination of the histone H2A (H2AK119ub1) which, in turn, affects the expression of genes related to neural tube closure [15]. The administration of FA stimulates pyrimidine and purine synthesis and the methylation of key macromolecules contributing to an early development of the nervous system [37]. In rats, it has been reported that the imbalance of nutrients (e.g. folates) in pregnant females showing normal or excess levels of FA leads to a reduction in the level of mRNAs/proteins (e.g. nerve growth factor, brain-derived neurotrophic factor) in the neonate brains [38]. In rodents, FA modulates cellular behavior in the nervous system, for example, generating chemotactic responses in the glia, inducing the release of neurotrophins and stimulating the cell differentiation of the PC-12 system [39]. It has been reported that a high metabolic rate of folates (including FA) does not allow their long-term storage in the body [40] and it seems that brainstem neurons metabolize FA during gestation periods (fetal and placental periods) allowing neurogenesis and neuroprotection, as well as avoiding the risk of malformations. FA and folate deficiencies have been related to neurodegenerative diseases and psychiatric disorders [17, 18, 20,41]. In this sense, using a murine model of Alzheimer's disease it has been reported that a dietary folate deficiency significantly increased homocysteine levels, which allowed a significant acceleration of the amyloidotic phenotype [18]. Moreover, in a mouse experimental model, it has been reported that a deficit in FA allowed a modulation of microRNAs (miR-106a-5p, miR200b-3p, miR-339-5p) associated with the accumulation of the amyloid precursor protein [17]. In rodents, a dietary FA supplementation promoted a neuroprotective effect and attenuated the severity of 6-OHDA-induced Parkinsonism [19] and in patients diagnosed with a first episode of schizophrenia, low folate and high homocysteine levels have been reported, compounds that can play an important role in the process of neurological development and in the clinical manifestation of this disorder [41]. Several studies have reported a relationship between FA deficiency and cerebral ischemia $[16$, 42]. Thus, in rodents with brain injury (after cerebral ischemia) and with a poor FA diet, the deficiency in this vitamin promoted an important injury in cortical neurons and the up-regulation of the LC3-II/LC3-I ratio and the expression of beclin-1 in these cells [16]. In addition, the combination of hypoxia-ischemia and dietary FA deficiency caused severe neural injury and a hippocampal microglial inflammatory response compared to hypoxia-ischemia alone [42]. In in vivo and in vitro experimental models, it has been reported that (after focal cerebral ischemia) a FA supplementation promoted the proliferation and migration of transplanted neural cell stem cells to the ischemic sites [14]. Finally, in a knockdown model of parkin (drosophila), the FA supplement produced a neuroprotective effect in dopaminergic neurons reducing motor disability, oxidative stress, adenosine triphosphate, and p53 [20]. 
In summary, the presence and distribution of cell bodies (fusiform, small/medium in size, showing one short dendrite) containing FA in the children brainstem have been described. It seems that the number of cell bodies decreases with age. This distribution was very restricted and immunoreactive perikarya were only observed in three regions of the pons. The distribution of FA in the central nervous system of humans and monkeys is different and, in addition, in these species FA was located in different parts of the nerve cells. In the children pons, the presence of FA suggests that the vitamin is involved in specific physiological mechanisms that must be elucidated in future studies.

\section{ORCID}

Ewing Duque-Díaz:

https://orcid.org/0000-0001-6306-8553

Rafael Coveñas: https://orcid.org/0000-0001-5677-8266

\section{Author Contributions}

Conceptualization: EDD, RC. Data acquisition: EDD, RC. Data analysis or interpretation: EDD, RC. Drafting of the manuscript: EDD, RC. Critical revision of the manuscript: EDD, RC. Approval of the final version of the manuscript: all authors.

\section{Conflicts of Interest}

No potential conflict of interest relevant to this article was reported.

\section{Acknowledgements}

This work has been supported by the Universidad de Santander UDES (PINLO/111702005-41823/EJ), Bucaramanga, Colombia and the "Programa XI: Programa de Financiación de Unidades de Excelencia de la Universidad de Salamanca", Salamanca, Spain. The authors want to thank the Morphophysiology Department (School of Medicine, Universidad de Santander, UDES) for kindly providing the human samples used in this work and the Language Service (University of Salamanca, Salamanca, Spain) for supervising the English text. The authors wish to thank Dr. Edwin Gómez Ramírez (Laboratory of Embryology and Physiology, Militar University Nueva Granada, Campus Cajicá, Colom- bia) for technical assistance.

\section{References}

1. Abdollahifar MA, Azad N, Sajadi E, Shams Mofarahe Z, Zare F, Moradi A, Rezaee F, Gholamin M, Abdi S. Vitamin C restores ovarian follicular reservation in a mouse model of aging. Anat Cell Biol 2019;52:196-203.

2. Hong JM, Kim JH, Kang JS, Lee WJ, Hwang YI. Vitamin C is taken up by human $\mathrm{T}$ cells via sodium-dependent vitamin $\mathrm{C}$ transporter 2 (SVCT2) and exerts inhibitory effects on the activation of these cells in vitro. Anat Cell Biol 2016;49:88-98.

3. Coveñas R, Mangas A, Bodet D, Duleu S, Marcos P, Karakas B, Geffard M. Frontiers in vitamin research: new antibodies, new data. ScientificWorldJournal 2011;11:1226-1242.

4. Coveñas R, Mangas A, Bodet D, Duleu S, Marcos P, Geffard M. Vitamin $\mathrm{C}$ in the monkey brain. In: Jackson CM, editor. Vitamin C: Nutrition, Side Effects, and Supplements. New York: Nova Science Publishers; 2011. p. 275-88.

5. Coveñas R, González-Fuentes J, Rivas-Infante E, LagartosDonate MJ, Mangas A, Geffard M, Arroyo-Jiménez MM, Cebada-Sánchez S, Insausti R, Marcos P. Developmental study of vitamin $\mathrm{C}$ distribution in children's brainstems by immunohistochemistry. Ann Anat 2015;201:65-78.

6. Mangas A, Coveñas R, Geffard K, Geffard M, Marcos P, Insausti R, Dabadie MP. Folic acid in the monkey brain: an immunocytochemical study. Neurosci Lett 2004;362:258-61.

7. Mangas A, Coveñas R, Geffard K, Geffard M, Marcos P, Insausti R, Glaize G, Dabadie MP. Riboflavin-like inmunoreactive fibers in the monkey brain. Anat Embryol (Berl) 2006;211:26772 .

8. Mangas A, Coveñas R, Geffard K, Geffard M, Marcos P, Insausti R, Dabadie MP. Thiamine-like fibers in the monkey brain: an immunocytochemical study. Life Sci 2006;79:1121-8.

9. Mangas A, Coveñas R, Bodet D, Duleu S, Marcos P, Geffard M. Vitamins in the monkey brain: an immunocytochemical study. J Chem Neuroanat 2009;38:1-8.

10. Mangas A, Bodet D, Duleu S, Yajeya J, Geffard M, Coveñas R. Direct visualization of retinoic acid in the rat hypothalamus: an immunohistochemical study. Neurosci Lett 2012;509:64-8.

11. Mangas A, Yajeya J, Gonzalez N, Husson M, Geffard M, Coveñas R. Detection of pantothenic acid-immunoreactive neurons in the rat lateral septal nucleus by a newly developed antibody. Folia Histochem Cytobiol 2016;54:186-192.

12. Chang S, Lu X, Wang S, Wang Z, Huo J, Huang J, Shangguan S, Li S, Zou J, Bao Y, Guo J, Wang F, Niu B, Zhang T, Qiu Z, Wu J, Wang L. The effect of folic acid deficiency on FGF pathway via Brachyury regulation in neural tube defects. FASEB J 2019;33:4688-702.

13. Li W, Ma Y, Li Z, Lv X, Wang X, Zhou D, Luo S, Wilson JX, Huang G. Folic acid decreases astrocyte apoptosis by preventing oxidative stress-induced telomere attrition. Int J Mol Sci 2019;21:62. 
14. Liu H, Cao J, Zhang H, Qin S, Yu M, Zhang X, Wang X, Gao Y, Wilson JX, Huang G. Folic acid stimulates proliferation of transplanted neural stem cells after focal cerebral ischemia in rats. J Nutr Biochem 2013;24:1817-22.

15. Pei P, Cheng X, Yu J, Shen J, Li X, Wu J, Wang S, Zhang T. Folate deficiency induced $\mathrm{H} 2 \mathrm{~A}$ ubiquitination to lead to downregulated expression of genes involved in neural tube defects. Epigenetics Chromatin 2019;12:69.

16. Zhao Y, Huang G, Chen S, Gou Y, Dong Z, Zhang X. Folic acid deficiency increases brain cell injury via autophagy enhancement after focal cerebral ischemia. J Nutr Biochem 2016;38:419.

17. Liu H, Tian T, Qin S, Li W, Zhang X, Wang X, Gao Y, Huang G. Folic acid deficiency enhances abeta accumulation in APP/PS1 mice brain and decreases amyloid-associated miRNAs expression. J Nutr Biochem 2015;26:1502-8.

18. Zhuo JM, Praticò D. Acceleration of brain amyloidosis in an Alzheimer's disease mouse model by a folate, vitamin B6 and B12-deficient diet. Exp Gerontol 2010;45:195-201.

19. Haghdoost-Yazdi H, Fraidouni N, Faraji A, Jahanihashemi H, Sarookhani M. High intake of folic acid or complex of B vitamins provides anti-Parkinsonism effect: no role for serum level of homocysteine. Behav Brain Res 2012;233:375-81.

20. Srivastav S, Singh SK, Yadav AK, Srikrishna S. Folic acid supplementation rescues anomalies associated with knockdown of parkin in dopaminergic and serotonergic neurons in Drosophila model of Parkinson's disease. Biochem Biophys Res Commun 2015;460:780-5.

21. Bremner JD, McCaffery P. The neurobiology of retinoic acid in affective disorders. Prog Neuropsychopharmacol Biol Psychiatry 2008;32:315-31.

22. Das BC, Thapa P, Karki R, Das S, Mahapatra S, Liu TC, Torregroza I, Wallace DP, Kambhampati S, Van Veldhuizen P, Verma A, Ray SK, Evans T. Retinoic acid signaling pathways in development and diseases. Bioorg Med Chem 2014;22:673-83.

23. Dev S, Adler AJ, Edwards RB. Adult rabbit brain synthesizes retinoic acid. Brain Res 1993;632:325-8.

24. Luo T, Wagner E, Crandall JE, Dräger UC. A retinoic-acid critical period in the early postnatal mouse brain. Biol Psychiatry 2004;56:971-80.

25. Kane MA, Chen N, Sparks S, Napoli JL. Quantification of endogenous retinoic acid in limited biological samples by LC/MS/ MS. Biochem J 2005;388(Pt 1):363-9.

26. Kane MA, Folias AE, Wang C, Napoli JL. Quantitative profiling of endogenous retinoic acid in vivo and in vitro by tandem mass spectrometry. Anal Chem 2008;80:1702-8.

27. Duque E, Mangas A, Salinas P, Díaz-Cabiale Z, Narváez JA, Coveñas R. Mapping of alpha-neo-endorphin- and neurokinin B-immunoreactivity in the human brainstem. Brain Struct Funct 2013;218:131-49.

28. Guntern R, Vallet PG, Bouras C, Constantinidis J. An improved immunohistostaining procedure for peptides in human brain. Experientia 1989;45:159-61.
29. Shi SR, Taylor CR. Standardization of immunohistochemistry based on antigen retrieval technique. In: Shi SR, Taylor CR, editors. Antigen Retrieval Immunohistochemistry Based Research and Diagnostics. Hoboken: John Wiley \& Sons; 2010. p. 87-99.

30. Coveñas R, González-Fuentes J, Rivas-Infante E, Lagartos-Donate MJ, Cebada-Sánchez S, Arroyo-Jiménez MM, Insausti R, Marcos P. Developmental study of the distribution of hypoxiainduced factor-1 alpha and microtubule-associated protein 2 in children's brainstem: comparison between controls and cases with signs of perinatal hypoxia. Neuroscience 2014;271:77-98.

31. Haines DE. Neuroanatomy: an atlas of structures, sections and systems. 2nd ed. Baltimore: Urban \& Schwartzenberg; 2012.

32. Broeke J, Mateos J, Pascau J. Image processing with ImageJ. 2nd ed. Birmingham: Packt Publishing; 2015.

33. Sánchez ML, Díaz-Cabiale Z, Narváez JA, Manso B, Salinas P, Rivada E, Smith V, Coveñas R. Mapping of methionineenkephalin-arg6-gly7-leu8 in the human diencephalon. Neuroscience 2016;334:245-58.

34. Coveñas R, Martin F, Belda M, Smith V, Salinas P, Rivada E, Diaz-Cabiale Z, Narvaez JA, Marcos P, Tramu G, GonzalezBaron S. Mapping of neurokinin-like immunoreactivity in the human brainstem. BMC Neurosci 2003;4:3.

35. Morse NL. Benefits of docosahexaenoic acid, folic acid, vitamin $\mathrm{D}$ and iodine on foetal and infant brain development and function following maternal supplementation during pregnancy and lactation. Nutrients 2012;4:799-840.

36. Dean JH, Pauly R, Stevenson RE. Neural tube defects and associated anomalies before and after folic acid fortification. J Pediatr 2020;226:186-94.E4.

37. Copp AJ, Adzick NS, Chitty LS, Fletcher JM, Holmbeck GN, Shaw GM. Spina bifida. Nat Rev Dis Primers 2015;1:15007.

38. Sable P, Dangat K, Kale A, Joshi S. Altered brain neurotrophins at birth: consequence of imbalance in maternal folic acid and vitamin $B_{12}$ metabolism. Neuroscience 2011;190:127-34.

39. Kim GB, Chen Y, Kang W, Guo J, Payne R, Li H, Wei Q, Baker J, Dong C, Zhang S, Wong PK, Rizk EB, Yan J, Yang J. The critical chemical and mechanical regulation of folic acid on neural engineering. Biomaterials 2018;178:504-16.

40. Radziejewska A, Chmurzynska A. Folate and choline absorption and uptake: their role in fetal development. Biochimie 2019;158:10-9.

41. Song X, Fan X, Li X, Kennedy D, Pang L, Quan M, Chen X, Gao J, Zhang W, Zhang J, Lv L. Serum levels of BDNF, folate and homocysteine: in relation to hippocampal volume and psychopathology in drug naïve, first episode schizophrenia. Schizophr Res 2014;159:51-5.

42. Cheng M, Yang L, Dong Z, Wang M, Sun Y, Liu H, Wang X, Sai N, Huang G, Zhang X. Folic acid deficiency enhanced microglial immune response via the Notch1/nuclear factor kappa B p65 pathway in hippocampus following rat brain I/R injury and BV2 cells. J Cell Mol Med 2019;23:4795-807. 